\title{
GASTRIC ATRESIA*
}

\author{
BY \\ H. WÜRTENBERGER \\ From the Surgical Department of the Children's Hospital, Bremen
}

In the newborn a complete occlusion of the pyloric outlet of the stomach is an extremely rare event. Very early vomiting occurs due to an absolute stoppage, but the vomitus contains no bile as found in stenosis or atresia below the ampulla of Vater. Respiratory troubles occur very quickly from breathing the overflowing stomach contents. The cause of stomach obstruction may be different. Pyloric spasm in the newborn is rare. Cystic masses in the gastric wall have been described.

\section{Case Report}

This case reports an intrinsic occlusion due to a membrane. The premature female infant, weighing 2,500 g., vomited mucus fluid on the second day after birth and later vomited her feeds; there was no bile, but meconium was passed. On admission at the age of 6 days her weight was $1,900 \mathrm{~g}$. A flat plane radiograph of the abdomen showed gas in the stomach, but none in the duodenum or the remainder of the small bowel (Fig. 1).

Despite the fact that the absence of gas in the rest of the abdomen is also typical of an atresia, operation was performed on the supposition of a pyloric spasm. We even found a very slight hypertrophy of the pyloric bulge and slit it following Weber-Ramstedt. But postoperatively the child continued vomiting and another radiograph showed there was no gas in the bowel. Three days later a further laparotomy had to be performed. The stomach was opened from a gastrotomy in the antral area. It was impossible to probe the pyloric channel because of a complete obstruction due to a solid membrane between antrum and pyloric channel (Fig. 2). After perforating the membrane and dilating the opening, a tube was inserted into the duodenum (Fig. 3). The passage was then free, the child could be fed through the tube and bowel movements were satisfactory. A further radiograph taken two weeks after the operation showed the opaque medium passing the pylorus beside the tube.

A short time later the child began vomiting again and the fluid residues in the stomach increased. It was decided at this third operation to perform a gastrojejunostomy. Postoperatively the passage through the anastomosis slowly began to work (Fig. 4). Nevertheless, the course was a dramatic one because of disturbances of

\footnotetext{
* A paper read at a meeting of the British Association of Paediatric Surgeons held in London in July 1960.
}

electrolytic balance as well as troubles from respiratory and circulatory systems. For a long time the child did not gain weight and only recently has there been a constant increase in weight.

\section{Discussion}

In the literature we have found only 10 cases of children operated upon in the first 12 days of life for a complete gastric occlusion. Bennett (1937), Touroff and Sussman (1940), and Fell (1951) found, as we did, the cause in a prepyloric diaphragm. Burnett and Halpert (1947) and Brown and Hertzler (1959) in other cases observed connective tissue up to $1 \mathrm{~cm}$. in length in the pyloric area, completely occluding the pyloric channel. A very interesting paper was given by Metz, Householder and DePree (1941). They found two membranes at a distance of $6 \mathrm{~cm}$. with a tumour palpable through the abdominal wall, containing gastric juice. From the 10 cases dealt with, five had been operated on successfully and re-examination showed free passage and a normal gastric function.

The cause of gastric atresia is almost unknown. In the foetal stage the stomach is at first a hollow organ. Until now no solid stage has been found, as is observed with the rest of the bowel in the fifth to tenth week of foetal life. Therefore an insufficient recanalization of solid stage as supposed in duodenal atresia cannot be the cause of gastric atresia. Touroff and Sussman believe that a fusion of the mucous membrane folds during the development of the pyloric bulges leads to the diaphragmatic occlusion.

In complete gastric obstruction indication for operation is clear. Pyloric spasm in the newborn rarely indicates complete absence of gas in the bowel; generally speaking, absence of gas in the bowel and vomitus without bile indicate a complete occlusion above the ampulla of Vater, either a membrane or atresia. Confusion with pyloric spasm is sometimes made and is described for instance by Bennett (1937).

The question remains as to what to do in atresia of the pyloric outlet of the stomach. An operative excision of a membrane by gastrotomy leads to 


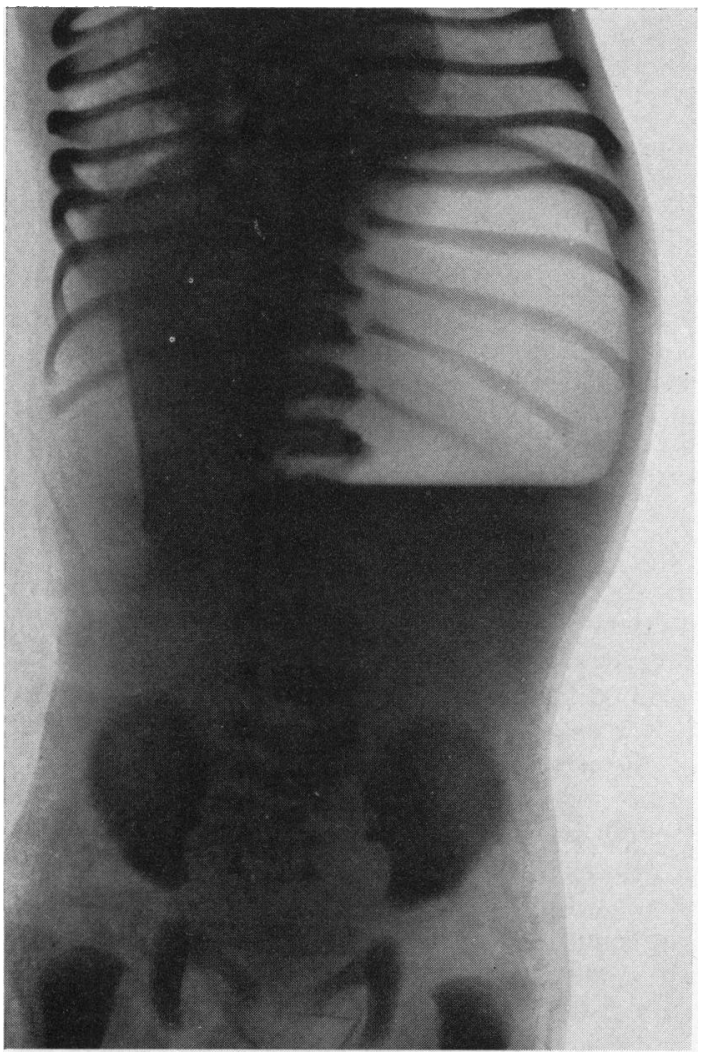

FIG. 1.

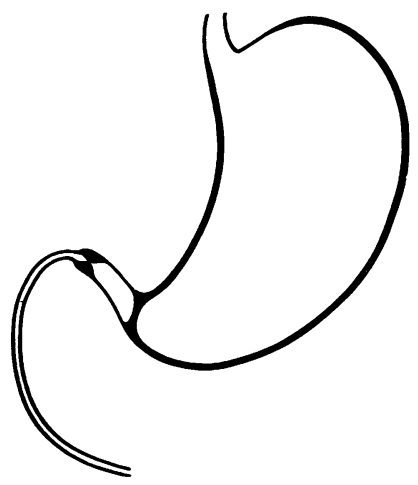

FiG. 2.

FIG. 1.-Antero-posterior radiograph, showing gaseous distension limited to the stomach.
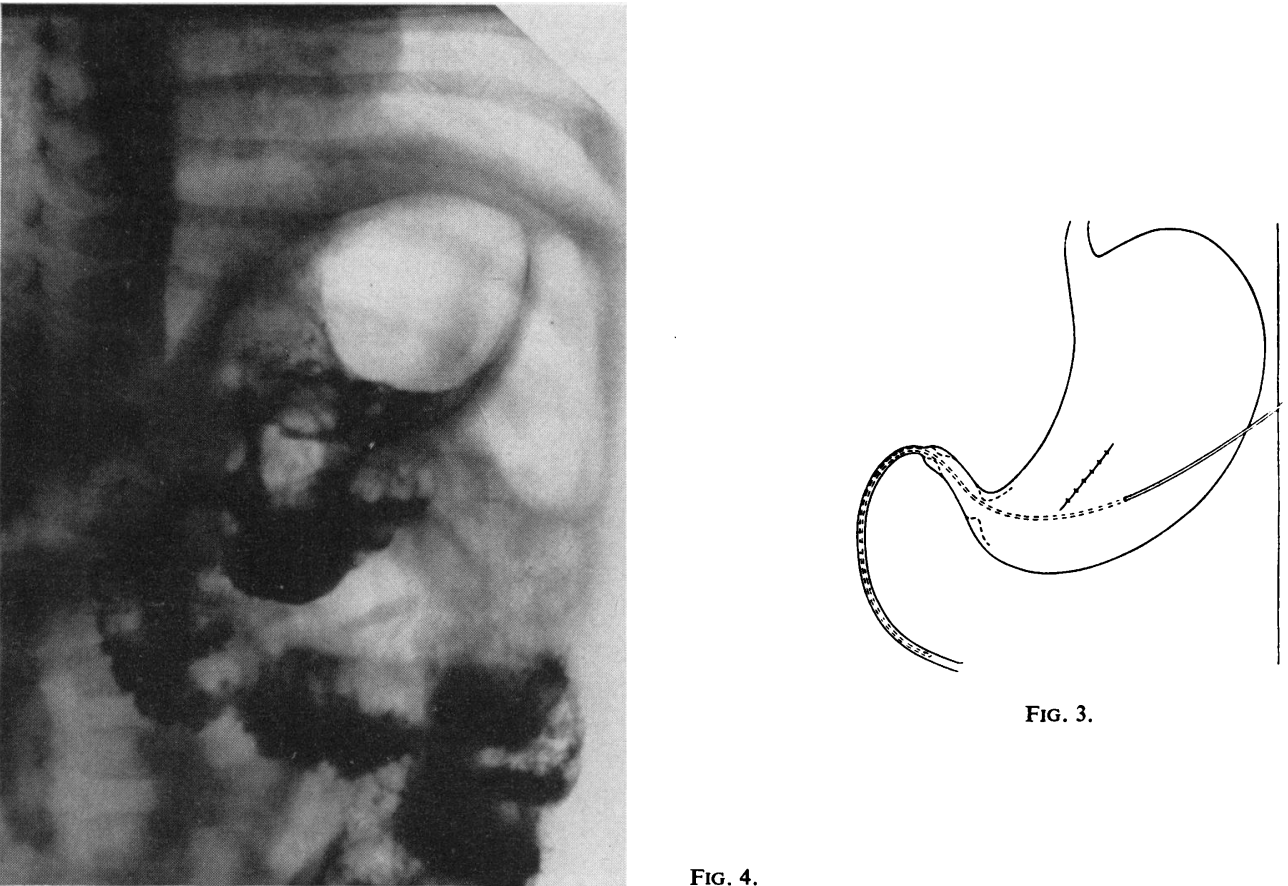

FIG. 2.-Diagram of the stomach with the intrinsic obstruction.

Fig. 3.-After opening the stomach in the antral area and perforating the membrane a tube was inserted into the duodenum.

Fig. 4.-Radiograph taken two weeks after performing the gastroenterostomy, showing satisfactory passage of the opaque medium into the small bowel.

Fig. 3.

Fig. 4. 
technical difficulties due to the considerable hypoplasia of the duodenum beyond the membrane. During excision damage to the wall may occur. To prevent this one can fill up the duodenum with sodium solution, so that the membrane will bulge slightly into the stomach. It is possible now to resect the central part and slit the border radially. Brown and Hertzler (1959) reported on two children with connective tissue $0.6 \mathrm{~cm}$. in length in the pyloric channel, found by a longitudinal incision of the pylorus. They opened the blind-ending sacks of mucous membrane and performed an anastomosis over a tube and finally closed the incision laterally.

We found quite different features in another case with a very small opening in the membrane. A 5-week-old child presented with a postpyloric membrane and we performed the excision without difficulty by gastrotomy. Unlike the child with complete occlusion, a great deal of air and fluid had run through the small opening; the duodenum and the distal bowel were functioning and therefore were of nearly normal calibre. This enlargement of calibre beyond the membrane considerably facilitates the excision and makes it fairly safe.

The perforation we tried seems questionable and cannot be more than a temporary help. Nevertheless, the perforation worked as a congenital opening, and led to improved bowel function facilitating the gastroenterostomy. There will not always be such good results as in the case of Benson and Coury (1951), who performed a gastroenterostomy after perforation and later found a free pyloric passage with closed anastomosis.

Because of the well-known disadvantages and dangers of a gastrojejunostomy, the decision to perform one is taken reluctantly. Three of the
10 children with gastroenterostomy died a few days after operation. One of these had a suture leakage and pneumonia and two others suffered from ileus, the exact cause for which was not known (Holladay 1946; Salzberg and Collins, 1960).

Possibly the complicated conditions of a gastrojejunostomy have been the cause of our child not progressing over a month. Until now there have been few reports of gastroenterostomy in the newborn, but the poor results in adults seem to prohibit its use in the newborn. What remains is pylorus resection or gastroduodenostomy, neither of which are particularly successful in the newborn infant. It is another question, whether we should use it in our case as a secondary step with removal of the gastroenterostomy after enlargement of the duodenum and small bowel and recovery by the child. In this way it would perhaps be possible to achieve somewhat normal conditions. On the other hand, such an intervention would not be simple or without risk in view of the fact that the child's abdomen has already been operated on three times.

REFERENCES

Bennett, R. J., Jr. (1937). Atresia of the pylorus. Amer.J. dig. Dis., $4,44$.

Benson, C. D. and Coury, J. J. (1951). Congenital intrinsic obstruction of the stomach and duodenum in the newborn. A.M.A. Arch. Surg., 62,856.

Brown, R. P. and Hertzler, J. H. (1959). Congenital prepyloric gastric atresia: a report of two cases. A.M.A.J. Dis. Child., 97,857 .

Burnett, H. A. and Halpert, B. (1947). Perforation of the stomach of a newborn infant with pyloric atresia. Arch. Path. (Chicago) 44. 318 .

Fell, E. H.(1951). In discussion on Benson and Coury (1951). A.M.A.

Arch. Surg., 62, 865.
Holladay, L. J. (1946). Case report of congenital aplasia of the pylorus. J. Indiana State med. Ass., 39, 350.

Metz, A. R., Householder, R. and DePree, J. F. (1941). Obstruction of the stomach due to congenital double septum with cyst formation. Trans, west. surg. Ass., 50, 242.

Salzberg, A. M. and Collins, R. E. (1960). Congenital pyloric atresia. A.M.A. Arch. Surg., 80, 501 .

Touroff, A.S. W. and Sussman, R. M. (1940). Congenital prepyloric membranous obstruction in a premature infant. Surgery, 